\title{
Comparison of initial tumor responses to transarterial bland embolization and drug-eluting beads-transarterial chemoembolization in the management of hepatocellular carcinoma: a propensity-score matching analysis
}

\author{
Jianxi Guo ${ }^{1 \#}$, Weidong Wang ${ }^{2 \#}$, Yanfang Zhang ${ }^{1}$, Linfeng $\mathrm{Xu}^{2}$, Jian Kong ${ }^{1 \wedge}$ \\ ${ }^{1}$ Department of Interventional Radiology, Shenzhen People's Hospital (Second Clinical Medical College of Jinan University, First Affiliated Hospital \\ of Southern University of Science and Technology), Shenzhen, China; ${ }^{2}$ Department of Interventional Radiology, Sun Yat-sen Memorial Hospital, \\ Sun Yat-sen University, Guangzhou, China \\ Contributions: (I) Conception and design: J Guo, W Wang, L Xu, J Kong; (II) Administrative support: Y Zhang, L Xu, J Kong; (III) Provision of study \\ materials or patients: J Guo, W Wang; (IV) Collection and assembly of data: J Guo, W Wang; (V) Data analysis and interpretation: J Guo, W Wang, \\ J Kong; (VI) Manuscript writing: All authors; (VII) Final approval of manuscript: All authors. \\ \#These authors contributed equally to this work and are co-first authors. \\ Correspondence to: Jian Kong. Department of Interventional Radiology, Shenzhen People's Hospital (Second Clinical Medical College of Jinan \\ University, First Affiliated Hospital of Southern University of Science and Technology), No. 1017, Dongmen Bei Road, Shenzhen 518020, China. \\ Email: drkkg@126.com; Linfeng Xu. Department of Interventional Radiology, Sun Yat-sen Memorial Hospital, Sun Yat-sen University, No. 107, \\ Yanjiang Xi Road, Guangzhou 510000, China. Email: xulf@mail.sysu.edu.cn.
}

Background: Transarterial bland embolization (TABE) is widely used to treat the spontaneous rupture of hepatocellular carcinoma (HCC), and can lead to ischemic necrosis of the tumor. In this study, we used the propensity-score matching (PSM) method to compare the initial responses of treatment-naïve HCC patients to TABE and drug-eluting beads-transarterial chemoembolization (DEB-TACE), and the safety of these treatments.

Methods: Patients with treatment-naïve HCC, who had been admitted to 2 medical centers from January 2016 to December 2020, were enrolled as the research subjects. The data of 26 patients treated with TABE for ruptured HCC and 52 patients treated with DEB-TACE for primary HCC were collected according to our inclusion and exclusion criteria, and a PSM analysis was conducted to assess the safety and effectiveness of these two interventional techniques 1 month postoperatively.

Results: In relation to ruptured HCC, TABE had a hemostatic success rate of $97.0 \%$. Before PSM, the TABE group had a larger maximum tumor diameter $(\mathrm{P}<0.05)$, a higher proportion of multiple tumors $(\mathrm{P}<0.05)$, a higher proportion of Child-Pugh class $\mathrm{B}(\mathrm{P}<0.05)$, and a higher proportion of Barcelona Clinic Liver Cancer (BCLC) stage B $(\mathrm{P}<0.05)$ than the DEB-TACE group. After PSM, the baseline characteristics of these two groups were well balanced, and there was no significant difference in patients' initial therapeutic responses and tumor recurrence rates (both $\mathrm{P}>0.05$ ). The multivariate regression analysis showed that tumor size was an independent predictor of the objective response rate (ORR) [odds ratio (OR): 3.312; 95\% CI: 0.152-5.944; $\mathrm{P}<0.05$ ]. Tumor number and BCLC stage also affected ORR; however, ORR was not significantly correlated with the interventional technique (TABE vs. DEB-TACE; P>0.05). The incidences of post-embolization syndrome (PES) and 48-h hepatotoxicity were significantly lower in the TABE group than the DEB-TACE group (both $\mathrm{P}<0.05$ ), but there was no significant difference in hepatotoxicity after 1 month $(\mathrm{P}>0.05)$.

$\wedge$ ORCID: 0000-0002-4786-2287. 
Conclusions: TABE is highly effective at managing hemorrhage from ruptured HCC. The initial therapeutic response of HCC to TABE is similar to that to DEB-TACE; however, TABE is associated with lower hepatotoxicity and fewer adverse effects, which paves the way for subsequent treatments and systemic therapies.

\author{
Keywords: Hepatocellular carcinoma (HCC); initial response; propensity-score matching (PSM); transarterial \\ bland embolization (TABE); drug-eluting beads-transarterial chemoembolization (DEB-TACE) \\ Submitted May 25, 2021. Accepted for publication Jul 26, 2021. \\ doi: 10.21037/jgo-21-370 \\ View this article at: https://dx.doi.org/10.21037/jgo-21-370
}

\section{Introduction}

Primary liver cancer (PLC) remains one of the most common malignancies worldwide, and it is the second leading cause of cancer-related death $(1,2)$. Approximately $90 \%$ of PLCs are hepatocellular carcinomas (HCCs) (3). Spontaneous rupture is a major life-threatening complication of HCC that occurs in $5-15 \%$ of cases, and is especially common in Asian countries where HCC is more frequent $(4,5)$. Patients may suffer from hemorrhagic shock if an HCC ruptures and bleeds, and the rate of acute mortality can reach $25-75 \%$ (6-9). Thus, the prompt and aggressive treatment of spontaneous rupture of HCC is particularly important.

HCC rupture often causes coagulation disorders and hemodynamic instability, and there is often insufficient time to precisely assess the hepatic reserve and tumor status when spontaneous rupture occurs. Thus, hemostasis is the primary goal of initial treatment. Transarterial bland embolization (TABE) or transarterial chemoembolization (TACE) can block the hepatic artery with embolic agents (e.g., iodine oil, gelatin sponge, and blank microspheres) under local anesthesia; thus, achieving rapid and definite hemostasis within a short period $(7,10)$ and avoiding the risk of liver failure after surgical and anesthetic procedures. In our clinical practice, we have found that the embolization of the hepatic artery with blank microspheres not only achieves successful hemostasis but also has certain therapeutic effects on HCC by the induction of ischemia and necrosis in the tumor area. However, the difference in any such anti-tumor effects between TABE and drug-eluting beads-transarterial chemoembolization (DEB-TACE) remains unclear.

In this study, we retrospectively analyzed the outcomes of patients treated with TABE for ruptured HCC at two centers in China and compared the therapeutic responses of treatment-naive HCC patients treated with DEB-TACE to identify the initial effectiveness and safety of TABE and
DEB-TACE in treating HCC. We present the following article in accordance with the STROBE reporting checklist (available at https://dx.doi.org/10.21037/jgo-21-370).

\section{Methods}

\section{Subjects}

This multicenter retrospective propensity-score-matched non-randomized controlled study was approved by the Medical Ethics Committee of Shenzhen People's Hospital (No. LL-KY-2021640). Informed consent has been attained from patients and his or her family before surgery, and informed consent for this retrospective study was waived. All procedures performed in this study involving human participants were in accordance with the Declaration of Helsinki (as revised in 2013).

\section{Patient selection}

Using an inpatient information retrieval system, we searched for patients with a primary diagnosis of HCC who had been treated at two medical centers between January 2016 and December 2020 (11-13). Patients in the TABE group experienced hemorrhage due to ruptured HCC following TABE (symptoms included acute abdominal pain and shock, positive shifting dullness during abdominal percussion, and non-coagulated blood during the diagnostic laparotomy; the diagnosis of spontaneous rupture of HCC was confirmed by ultrasound, CT, and tumor markers). Patients in the DEBTACE group were inoperable or refused surgical treatment and treated with DEB-TACE. Patients in both groups were enrolled for propensity-score matching (PSM) based on our inclusion and exclusion criteria (see below).

To be eligible to participate in this study, patients had to meet the following inclusion criteria: (I) be aged $\geq 18$ years; (II) have a blood creatinine level $<1.2 \mathrm{mg} / \mathrm{dL}$, 
ASL/ALT <200 IU/L, and total bilirubin $<3 \mathrm{mg} / \mathrm{dL}$; (III) have liver function Child-Pugh class A or B and Barcelona Clinic Liver Cancer (BCLC) stage A or B, be inoperable or refuse surgical treatment, and meet the indications for TABE or DEB-TACE; (IV) have single or multiple tumors (with the diameter of the major lesion $\geq 1 \mathrm{~cm}$ ), and have $\leq 60 \%$ affected liver parenchyma; (V) have not received any other treatment (e.g., surgical resection, intervention, chemotherapy, radiotherapy, targeted therapy, or immunotherapy) for HCC before the initial TABE/DEBTACE treatment and the first imaging follow-up; and (VI) not have complete occlusion of the main trunk of the portal vein, extrahepatic metastasis, or large vessel invasion.

Patients were excluded from the study if they met any of the following exclusion criteria: (I) had received other treatment(s) (e.g., surgical resection, intervention, chemotherapy, radiotherapy, targeted therapy, and/ or immunotherapy) for HCC before the initial TABE/ DEB-TACE treatment or the first imaging follow-up; (II) had secondary HCC or diffuse HCC; (III) had complete occlusion of the portal vein trunk by cancer thrombus and/or obvious arteriovenous fistulas identified during interventional procedures ; (IV) were lost during follow-up or were unable to attend the follow-up visits; (V) had coexisting severe insufficiency of heart, lungs, liver, and/or kidneys; (VI) had contraindications (e.g., severe coagulation dysfunction and/or history of iodine allergy) to vascular intervention; and/or (VII) were a pregnant or lactating female.

\section{Data collection}

Basic patient information (including age, sex, past medical histories, personal history, current medical histories, and treatment histories), systemic conditions (including consciousness, heart rate, and blood pressure), imaging findings [including abdominal ultrasonography (US), computed tomography (CT)/magnetic resonance imaging (MRI), and angiography], laboratory test results [including routine blood tests, coagulation function, liver, and kidney functions, testing for infectious diseases, and tumor markers, such as alpha-fetoprotein (AFP)] at admission and before and after TABE/DEB-TACE treatment, tumor status (including tumor number, location, and size), and interventions (the techniques and outcomes) were collected.

\section{Treatment}

All the interventional procedures were performed under digital subtraction angiography (DSA) $(1,200 \mathrm{~mA}$, Siemens Medical Solutions, Munich, Germany) guidance by operators who had completed interventional specialty training and had more than 10 years' independent operation experience. Each patient was placed in a supine position. The bilateral groin areas were routinely disinfected and draped. After local anesthesia, the right femoral artery was accessed with a $5 \mathrm{~F}$ arterial sheath (Terumo Co., Tokyo, Japan) using the Seldinger technique. A 5F Pigtail catheter (Cordis, Miami Lakes, USA) was introduced under the guidance of a guidewire (Terumo, Tokyo, Japan) for abdominal aortography to observe any variant and/ or parasitic vessels supplying the tumors. Subsequently, a Yashiro catheter (Terumo, Tokyo, Japan) was placed in the abdominal trunk or common hepatic artery for acquisition of a set of volumetric images at arterial, venous, and parenchymal phases. The collateral arteries supplying HCC were searched, and the tumor blood supply, portal venous blood flow, and co-existing hepatic arteriovenous fistulae (if any) were assessed. A 2.4F coaxial microcatheter (Boston Scientific, Boston, USA) was ultra-selectively placed in the segment or subsegment of the tumor-supplying artery, avoiding the gallbladder artery and hepatic falciform artery (HFA). An embolic agent was then injected to cut off blood flow. After the procedure was completed, the catheters and sheaths were removed. Pressure was held at the site of the puncture to stop bleeding. The right lower extremity was immobilized for 12 hours. The routine postoperative symptomatic treatments included hepatoprotection, gastric protection, analgesia, and the use of antiemetics.

\section{TABE group}

After the application of fluid resuscitation to maintain hemodynamic stability and after informed consent had been obtained from the patient and his/her family members, an appropriate amount and size of blank microspheres (8 Spheres ${ }^{\circledR}$, Hengrui Medical, Suzhou, China) were used according to each patient's liver function status, systemic condition, tumor burden (tumor size and number of lesions), and intraoperative angiographic findings. Gelatin sponge particles (Alicon, Hangzhou, China), 1,000-1,400 microm in diameter, were also used if necessary. The predefined endpoint of the embolization was the complete disappearance of the main tumor-feeding arteries and the tumor stain.

\section{DEB-TACE group}

The most commonly selected particle sizes of the drug- 
eluting microspheres were 100-300 $\mu \mathrm{m} .2 \mathrm{~mL}$ of drugeluting microspheres (CalliSpheres ${ }^{\circledR}$, Hengrui Medical, Suzhou, China) was added with $50 \mathrm{mg}$ of pirarubicin. After the drug was loaded, $10-15 \mathrm{~mL}$ of ioversol 350 (Hengrui Medical, Suzhou, China) was added. An appropriate volume of sterilized water for injection and contrast agent was added to obtain homogeneous suspension. An appropriate amount of drug-eluting microspheres were selected for embolization according to the tumor size, number of lesions, and intraoperative angiographic findings. The predefined endpoint of the embolization was the complete disappearance of the tumor stain and the presence of the "tree-in-the winter" in the main tumor-feeding arteries. The contrast agent was cleared after 2-5 cardiac cycles.

\section{Follow-up}

All patients underwent a plain and enhanced abdominal CT or MRI scan at 1 month postoperatively, and the images were interpreted by two experienced radiologists blind to the treatment protocol or clinical information. Disagreements were solved by negotiation. Response evaluation was performed using the modified Response Evaluation Criteria in Solid Tumors (mRECIST) (14), which defines tumor response as complete response (CR), partial response (PR), stable disease (SD), or progressive disease $(\mathrm{PD})$. Initial response was defined as tumor changes, including $\mathrm{CR}, \mathrm{PR}, \mathrm{SD}$, and $\mathrm{PD}$, as assessed at imaging 1 month after the first TABE or DEB-TACE session (15). The objective response rate (ORR) was calculated using the following formula: $\mathrm{ORR}=(\mathrm{CR}+\mathrm{PR}) /$ total number of cases $\times 100 \%$. All patients were closely monitored and underwent clinical examinations, laboratory tests, and tumor evaluations. Adverse postoperative reactions were accorded according to the Common Terminology Criteria for Adverse Events (CTCAE) version 3.0. A subsequent treatment plan was developed according to the BCLC algorithm and Chinese NCLC guidelines.

\section{Endpoints}

The primary endpoint of our current study was the effectiveness of TABE or DEB-TACE in treating tumors. The effectiveness was the initial response rate (e.g., ORR) after 1 month of TABE or DEB-TACE, and was assessed using the mRECIST criteria.

The two secondary endpoints were as follows: (I) the safety of the TABE or DEB-TACE for HCC. All adverse events (AEs) were assessed and recorded. The primary safety endpoint was hepatotoxicity, as assessed by bilirubin and AST/ALT changes at $48 \mathrm{~h}$ and 1 month postoperatively, and the secondary safety endpoint was the incidence and grade of post-embolization syndrome (PES); and (II) hemostatic efficacy, which was assessed using the hemostatic success rate of TABE for ruptured HCC. "Hemostatic success" was defined as: (i) stable vital signs after transhepatic artery embolization; (ii) no active bleeding within 1 month after embolization; and (iii) no significant hemoglobin and hematocrit changes at two postoperative reexaminations.

\section{Statistical analysis}

Statistical analyses were conducted using SPSS 13.0 software package (IBM Corp., Armonk, NY, USA) and $\mathrm{R}$ programming language. The normally distributed continuous variables are expressed as mean \pm standard deviation (SD), while the non-normally-distributed continuous variables are expressed as median (interquartile range). The count data are presented as $\mathrm{n}(\%)$. The measurement data were compared using a $t$ test if they were normally distributed, or a Mann-Whitney $\mathrm{U}$ test otherwise. Categorical variables were compared using a Chi Squaretest with Yates correction or Fisher's exact test (for small samples). The logistic multivariate analyses were performed before and after PSM to correct for differences in patient variables between the two groups to identify independent risk factors affecting the outcomes. A $\mathrm{P}$ value of $<0.05$ was considered statistically significant.

The following variables were included in the PSM: age, gender, type of cirrhosis (HBV, HCV, alcoholism, and other), tumor status (size, number, and location), ChildPugh class, BCLC stage, and AFP. The caliper was set to 0.2 standard deviations to achieve optimal homogeneity and minimal sample loss.

\section{Results}

\section{Baseline clinical characteristics of patients}

In total, 726 patients with treatment-naïve HCC who had been admitted to two medical centers from January 2016 to December 2020 were enrolled as research subjects. Among them, 67 were diagnosed with spontaneous rupture of HCC and underwent TABE. Of these 67 patients, 46 were male $(68.7 \%)$ and 21 were female $(31.3 \%)$. The patients 
Table 1 Baseline characteristics of all patients undergoing TABE for spontaneous rupture of HCC

\begin{tabular}{lc}
\hline Parameters & TABE $(\mathrm{n}=67)$ \\
\hline Gender (male/female) & $46 / 21$ \\
Age (years) & $57.2(12.3)$ \\
Etiology (HBV/HCV/alcoholism/other) & $47 / 11 / 7 / 2$ \\
Maximum tumor diameter (cm) & $9.8(5.5)$ \\
$\leq 6 />6$ & $28 / 39$ \\
Single/multiple tumors & $19 / 48$ \\
Location (right lobe/left lobe/bilobed) & $34 / 13 / 20$ \\
Child-Pugh class (A/B/C) & $25 / 38 / 4$ \\
BCLC grade (A/B/C/D) & $19 / 32 / 12 / 4$ \\
AFP (ng/mL) & \\
$<200 / \geq 200$ & $16 / 51$ \\
Initial symptoms (abdominal pain/ & $49 / 7 / 11$ \\
distension/shock)
\end{tabular}

TABE, transarterial bland embolization; HCC, hepatocellular carcinoma; BCLC, Barcelona Clinic Liver Cancer; AFP, alphafetoprotein.

had an average age of $57.2 \pm 12.3$ years (range, $24-77$ years). Cirrhosis was caused by HBV infection in 47 cases (70.1\%), HCV infection in 11 cases (16.4\%), and alcoholism/other in 9 cases $(13.4 \%)$. The mean maximum tumor diameter was $9.8 \pm 5.5 \mathrm{~cm}$, and 48 patients $(71.6 \%)$ had multiple lesions. The Child-Pugh class was A in 25 cases (37.3\%), $\mathrm{B}$ in 38 cases $(56.7 \%)$, and $\mathrm{C}$ in 4 cases $(6.0 \%)$. The BCLC stage was A in 19 cases $(28.4 \%)$, B in 32 cases $(47.8 \%)$, $\mathrm{C}$ in 12 cases $(17.9 \%)$, and $\mathrm{D}$ in 4 cases $(6.0 \%)$. Among the 67 patients, 52 received TABE within $24 \mathrm{~h}$ of tumor rupture, and 15 received embolization within 1 week of tumor rupture. Tumors rich in blood supply were found on the hepatic angiographies of all patients. Contrast media extravasation (CMEV) was observed in 11 patients (16.4\%), which suggested the presence of active bleeding. Patients' initial symptoms included sudden abdominal pain $(\mathrm{n}=49,73.1 \%)$, abdominal distension $(\mathrm{n}=7,10.4 \%)$, and hypovolemic shock ( $\mathrm{n}=11,16.4 \%$; see Table 1$)$. After TABE was performed to treat bleeding due to ruptured HCC, 6 patients died during the 1-month follow-up period; their causes of death included liver failure $(\mathrm{n}=2)$, variceal bleeding $(\mathrm{n}=1)$, hepatorenal syndrome $(\mathrm{n}=1)$, and bleeding due to HCC re-rupture $(n=2)$. Thus, TABE had a hemostatic success rate of $97.0 \%(65 / 67) .61$ patients continued to receive follow-up and subsequent treatments after 1 month, and 3 of these patients underwent surgical resection.

Based on the inclusion and exclusion criteria, 24 patients were excluded for one of the following reasons: (I) they had received anti-tumor treatments other than TABE/TACE before rupture and during the follow-up period; (II) they had died or were lost during the follow-up period within 1 month of the intervention; (III) they had a Child-Pugh class of C; (IV) they had a BCLC stage of C or D; and/or (V) more than $60 \%$ of their hepatic parenchyma was affected. Thus, 43 patients entered the final analysis. Additionally, 105 patients with primary HCC treated with DEB-TACE were retrieved from the literature. 1:2 PSM yielded 26 patients undergoing TABE for spontaneous rupture of HCC, and 52 matched patients with primary HCC treated with DEB-TACE. The flowchart of patient enrollment is shown in Figure 1.

\section{Comparison of baseline characteristics before and after PSM}

Before PSM, there were significant differences in the baseline characteristics between the TABE and DEB-TACE groups. More specifically, before PSM, the TABE group had a longer maximum tumor diameter (mean: 10.3 vs. $7.5 \mathrm{~cm} ; \mathrm{P}<0.05)$, a higher proportion of multiple tumors (58.1\% vs. $38.1 \% ; \mathrm{P}<0.05)$, a higher proportion of ChildPugh class B $(65.1 \%$ vs. $30.5 \%$; $\mathrm{P}<0.05)$, and a higher proportion of BCLC stage $\mathrm{B}(58.1 \%$ vs. $35.2 \%$; $\mathrm{P}<0.05)$ than the DEB-TACE group (see Table 2). Thus, PSM was necessary. As described above, a 1:2 PSM yielded 26 patients undergoing TABE for hemorrhage from ruptured primary HCC and 52 matched patients with primary HCC treated with DEB-TACE. The baseline characteristics were no longer significant between the TABE and DEB-TACE groups after PSM (see Table 3).

\section{Postoperative complications and hepatotoxicity associated with TABE or DEB-TACE}

All patients underwent the intervention as planned, and neither major complications nor grade 3-4 toxicities occurred within 1 month of the procedure. Most patients experienced mild PES after the procedure, including nausea/vomiting, fever unrelated to infection, and mild to moderate abdominal pain. The incidence of PES was significantly lower in the TABE group than in the DEB- 


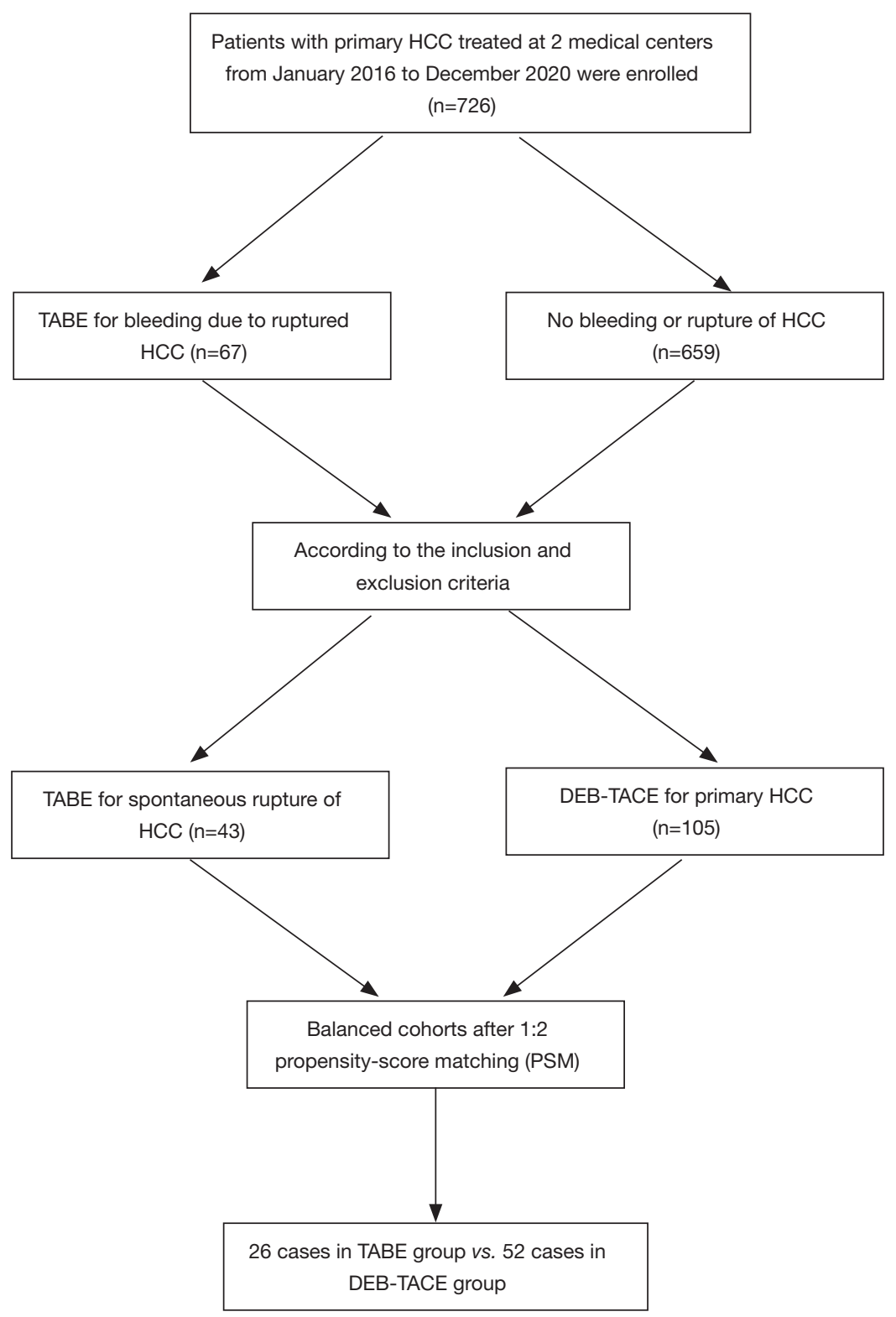

Figure 1 The flowchart of patient enrollment.

TACE group $(\mathrm{P}<0.05)$. Complications, such as intrahepatic bile duct dilatation and cholecystitis, were not significantly different between the two groups $(\mathrm{P}>0.05)$ and were alleviated by pharmacological treatment. No patient developed liver abscess, bilomas, or pancreatitis. The incidence of 48-h hepatotoxicity was also significantly lower in the TABE group than in the DEB-TACE group $(\mathrm{P}<0.05)$ but did not significantly differ after 1 month $(\mathrm{P}>0.05$; see Table 4).

\section{Initial response and tumor recurrence rate at 1 month after TABE or DEB-TACE}

Patients' initial response at 1 month after procedure was evaluated by CT or MRI (see Table 5). In the TABE group, there were 0 cases of CR ( $0 \%), 15$ cases of PR (57.7\%), 6 cases of SD $(23.1 \%)$, and 5 cases of PD $(19.2 \%)$. In the DEB-TACE group, there were 0 cases of CR $(0 \%), 34$ cases of PR (65.4\%), 11 cases of SD (19.2\%), and 7 cases of PD (13.5\%). The ORR did not differ significantly between the 
Table 2 Baseline data of TABE and DEB-TACE groups before PSM

\begin{tabular}{|c|c|c|c|c|}
\hline Parameters & TABE $(n=43)$ & DEB-TACE $(n=105)$ & $\mathrm{T} / \chi^{2} / Z$ values & $P$ value \\
\hline Age (years) & $51.2(15.8)$ & $52.6(17.1)$ & 0.571 & 0.647 \\
\hline Etiology (HBV/HCV/alcoholism/other) & $31 / 7 / 5 / 0$ & $76 / 17 / 8 / 4$ & 0.016 & 0.987 \\
\hline Maximum tumor diameters (cm) & $10.3(6.1)$ & $7.5(5.3)$ & 0.393 & 0.007 \\
\hline Single/multiple tumors & $18 / 25$ & $65 / 40$ & 4.976 & 0.026 \\
\hline Location (right lobe/left lobe/bilobed) & $23 / 9 / 11$ & $52 / 23 / 30$ & 0.204 & 0.651 \\
\hline Child-Pugh class A/B & $15 / 28$ & $73 / 32$ & 15.186 & 0.000 \\
\hline$B C L C$ grade $A / B$ & $18 / 25$ & $68 / 37$ & 6.573 & 0.010 \\
\hline
\end{tabular}

TABE, transarterial bland embolization; DEB-TACE, drug-eluting beads-transarterial chemoembolization; PSM, propensity-score matching; BCLC, Barcelona Clinic Liver Cancer.

Table 3 Baseline data of TABE and DEB-TACE groups after PSM

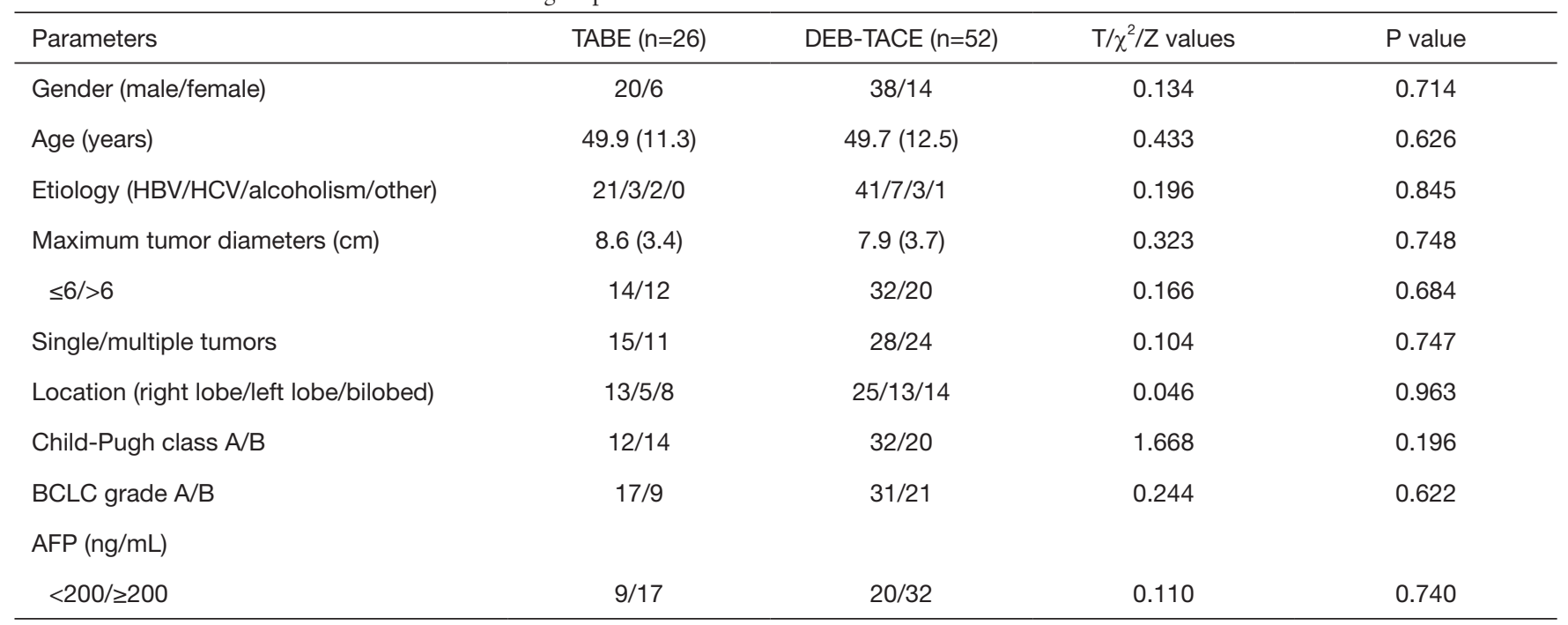

TABE, transarterial bland embolization; DEB-TACE, drug-eluting beads-transarterial chemoembolization; PSM, propensity-score matching; BCLC, Barcelona Clinic Liver Cancer.

TABE and DEB-TACE groups $\left(57.7 \%\right.$ vs. $65.4 \% ; \chi^{2}=0.439$, $\mathrm{P}=0.508)$.

In the TABE group, local recurrence occurred in 3 cases, and new lesions were detected in 2 cases. In the DEBTACE group, local recurrence occurred in 3 cases, and new lesions were detected in 4 cases. Thus, the recurrence rate was not significantly different between the TABE and DEBTACE groups ( $13.5 \%$ vs. $\left.19.2 \% ; \chi^{2}=0.352 ; \mathrm{P}=0.553\right)$.
In the TABE group, there were 17 patients with baseline AFP levels of $\geq 200 \mathrm{ng} / \mathrm{mL}, 0$ patients with a normalized AFP level after TABE treatment, and 9 patients (52.9\%) with an AFP reduction of $\geq 50 \%$ relative to the baseline. In the DEB-TACE group, there were 33 patients with baseline AFP levels of $\geq 200 \mathrm{ng} / \mathrm{mL}, 0$ patients with a normalized AFP level after DEB-TACE treatment, and 19 patients $(57.6 \%)$ with an AFP reduction of $\geq 50 \%$ relative to the 
Table 4 Postoperative complications and hepatotoxicity associated with TABE or DEB-TACE after PSM

\begin{tabular}{|c|c|c|c|c|}
\hline Parameters & TABE $(n=26)$ & DEB-TACE $(n=52)$ & $\chi^{2} / Z$ values & $P$ value \\
\hline $\begin{array}{l}\text { Post-embolization syndrome (e.g., } \\
\text { nausea/vomiting, abdominal pain, and } \\
\text { fever) }\end{array}$ & $13(50.0 \%)$ & $39(75.0 \%)$ & 4.875 & 0.027 \\
\hline Intrahepatic bile duct dilatation & $1(3.8 \%)$ & $5(9.6 \%)$ & 0.203 & 0.652 \\
\hline \multicolumn{5}{|l|}{ 48-h hepatotoxicity } \\
\hline Bilirubin (grade 1/2/3) & $7 / 2 / 1$ & $23 / 7 / 5$ & 2.119 & 0.034 \\
\hline AST (grade 1/2/3/4) & $10 / 7 / 6 / 0$ & $34 / 11 / 5 / 1$ & 2.480 & 0.013 \\
\hline ALT (grade 1/2/3/4) & $7 / 4 / 3 / 1$ & $22 / 14 / 9 / 0$ & 2.397 & 0.017 \\
\hline AST (grade 1/2) & $7 / 2$ & $23 / 3$ & 1.402 & 0.161 \\
\hline ALT (grade 1/2) & $5 / 3$ & $19 / 4$ & 1.339 & 0.181 \\
\hline
\end{tabular}

TABE, transarterial bland embolization; DEB-TACE, drug-eluting beads-transarterial chemoembolization; PSM, propensity-score matching; AST, aspartate aminotransferase; ALT, alanine aminotransferase.

Table 5 Comparison of patients' initial responses at 1 month postoperatively between the TABE and DEB-TACE groups after PSM

\begin{tabular}{lcccccc}
\hline \multirow{2}{*}{ Group } & $\mathrm{n}$ & \multicolumn{4}{c}{ mRECIST } \\
\cline { 3 - 5 } & & $\mathrm{CR}$ & $\mathrm{PR}$ & $\mathrm{SD}$ & $\mathrm{PD}$ & \multicolumn{1}{c}{ ORR } \\
\hline TABE & 26 & 0 & $15(57.7 \%)$ & $6(23.1 \%)$ & $5(19.2 \%)$ & $15(57.7 \%)$ \\
DEB-TACE & 52 & 0 & $34(65.4 \%)$ & $11(19.2 \%)$ & $7(13.5 \%)$ & $34(65.4 \%)$ \\
$\chi^{2}$ value & - & - & 0.439 & 0.038 & 0.352 & 0.439 \\
$P$ value & - & - & 0.508 & 0.846 & 0.553 & 0.508 \\
\hline
\end{tabular}

TABE, transarterial bland embolization; DEB-TACE, drug-eluting beads-transarterial chemoembolization; PSM, propensity-score matching.

baseline. Thus, there was no significant difference in the AFP reduction between the TABE and DEB-TACE groups (52.9\% vs. $\left.57.6 \% ; \chi^{2}=0.098 ; \mathrm{P}=0.754\right)$.

The univariate analysis showed that the following 4 prognostic factors affected tumor response: tumor size ( $\leq 6 v s .>6 \mathrm{~cm}$ ), the number of lesions (single $v s$. multiple), BCLC stage (A vs. B), and AFP level (<200 vs. $\geq 200)$. The multivariate analysis showed that tumor size was an independent predictor of ORR (OR: 3.312, 95\% CI: $0.152-$ 5.944; $\mathrm{P}<0.05)$. In addition, the number of lesions and BCLC stage also affected ORR; however, the initial ORR was not significantly correlated with treatment protocol (OR: 0.818, 95\% CI: 0.636-5.619; P>0.05; see Table 6).

\section{Discussion}

PLC is a common cancer worldwide. Due to the high prevalence of $\mathrm{HBV}$ infection, over half of the cases of HCC worldwide occur in China (16). The incidence of spontaneous rupture of HCC is low in Western countries (about $3 \%$ ), but can be as high as $15 \%$ in Eastern countries (4-6,17). The current tumor, node, and metastasis (TNM) systems directly classify ruptured HCC as T4 when no other clinical indicator of the tumor is considered $(18,19)$. However, the result of such a TNM classification is controversial, as it does not accurately reflect the real-world prognosis of a ruptured HCC $(9,20)$. The TNM-based Japan Integrated Staging score (21) was not feasible for 
Table 6 Univariate and multivariate analyses of the predictors of ORR after PSM

\begin{tabular}{|c|c|c|c|c|}
\hline Parameters & Univariate ( $P$ value) & Multivariate (P value) & $\operatorname{Exp}(B)$ & $95 \% \mathrm{Cl}$ \\
\hline Gender (male/female) & 0.553 & 0.928 & 0.608 & $0.215-24.833$ \\
\hline Child-Pugh class (A/B) & 0.378 & 0.132 & 1.809 & $0.371-3.159$ \\
\hline Tumor diameter $(\leq 6 />6 \mathrm{~cm})$ & 0.004 & 0.006 & 3.312 & $0.152-5.944$ \\
\hline $\begin{array}{l}\text { Tumor location (right lobe/left lobe/ } \\
\text { bilobed) }\end{array}$ & 0.055 & 0.487 & 1.561 & $0.574-4.018$ \\
\hline BCLC stage (A/B) & 0.031 & 0.047 & 1.286 & $0.089-5.956$ \\
\hline AFP level $(<200 / \geq 200 \mathrm{ng} / \mathrm{dL})$ & 0.028 & 0.439 & 1.357 & $1.004-11.137$ \\
\hline
\end{tabular}

ORR, objective response rate; PSM, propensity-score matching; BCLC, Barcelona Clinic Liver Cancer; AFP, alpha-fetoprotein.

our current study, and thus we adopted the BCLC staging system, which is currently the most commonly used clinical staging system for PLC (22).

After admission, adequate resuscitation and hemodynamic stabilization were achieved by adjuvant therapies, such as intravenous therapies, blood transfusion, and the correction of coagulation disorders. TABE is the preferred method to achieve hemostasis (23). The success rate reported in the literature is as high as $53-100 \%$ $(6,23,24)$. In our current study, a 2 -stage management protocol was adopted to treat ruptured HCC. In stage I, an early and aggressive hemostatic intervention (mainly TABE) was performed to promote rapid and stable hemostasis and prevent tumor re-rupture. In stage II (the follow-up period), treatment protocols were developed according to the HCC diagnosis and treatment guidelines. Six of the 67 patients $(10.5 \%)$ died within 30 days of TABE with blank microspheres for ruptured HCC, and only two patients died from tumor re-rupture. Thus, the clinical success rate of hemostasis by TABE was 97.0\% (65/67), which is similar to the rates reported in literature. During stage II (follow-up) and as confirmed by the pathological studies of liver specimens, most of the HCC tissues showed necrotic changes after TABE (see Figure 2). Thus, both TABE and DEB-TACE were shown to have anti-tumor effects (see Figure 3); however, the differences between these two techniques require further investigation.

Patients with HCC frequently develop resistance to current chemotherapeutic drugs, which may be attributed to the expressions of multi-drug resistance genes and the abnormal p53 gene function $(25,26)$. In TACE therapy, the anti-tumor effects of embolic agents have been confirmed (27-29); however, it is unclear whether better anti-tumor efficacy can be obtained after the loading of chemotherapeutic drugs (30).

In recent years, a variety of embolic microspheres that can carry chemotherapeutic agents have been marketed in China and abroad, which appear to be able to be mixed with chemotherapeutic agents to release the drugs continuously and slowly (31). The 2018 NCCN Guidelines for Hepatobiliary Cancers list DEB-TACE as a recommended treatment for HCC (32). However, no guidelines recommend TABE for the treatment of HCC, and few controlled studies have explored the safety and efficacy of TABE for HCC or compared its effectiveness with DEB-TACE. Further, the use of TACE to treat HCC is mostly palliative; thus, most patients have already undergone multiple cycles of TACE or a combination of multiple regimens. These confounding factors make it particularly difficult to compare the efficacy of TABE with that of DEB-TACE directly. Thus, in our current study, only HCC patients who were treated with TABE or DEBTACE for the first time were included as research subjects, and patients who had received other treatments for the tumor before or during the study period were excluded.

We performed a PSM analysis to adjust for any baseline differences between both groups to compare patients' initial response of $\mathrm{HCC}$ to TABE or DEB-TACE in terms of effectiveness and safety in a more objective and accurate manner. Before PSM, there were significant differences in the baseline characteristics between the TABE and DEBTACE groups, which was subject to selection bias. Clearly, 

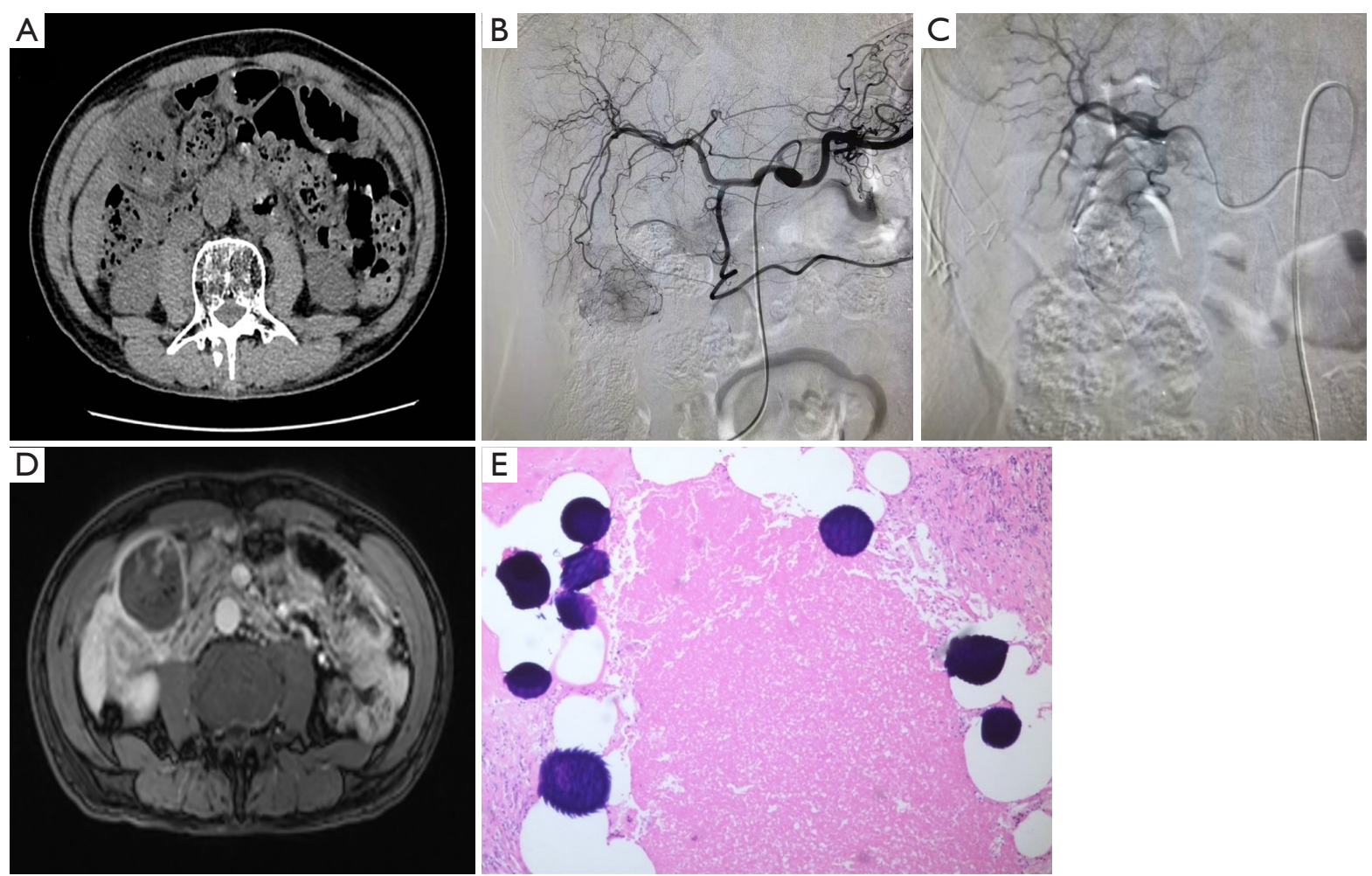

Figure 2 A 56-year-old male patient, with a previous history of hepatitis B for more than 20 years, presented at our emergency department with a sudden onset of abdominal pain. (A) The CT findings were suggestive of HCC rupture and bleeding; (B,C) an emergency TABE was performed to stop the bleeding; (D) A MRI scan performed 1 month after TABE revealed that most of the HCC lesions were significantly necrotic with a small number of contrast-enhanced tumor foci; (E) surgical pathology confirmed that most parts of the section were necrotic tissues with embolic microspheres visible in the vessels $(\mathrm{H} \& \mathrm{E}$, original magnification $\times 100)$. TABE, transarterial bland embolization; HCC, hepatocellular carcinoma.

it was inappropriate to directly compare the efficacy of these two intervention techniques without matching. Thus, the PSM (1:2) method was used to reduce the differences between the two groups. Notably, after PSM, the baseline characteristics showed no significant differences between the TABE and DEB-TACE groups.

Our response evaluation was based on patients' initial response 1 month after TABE or DEB-TACE. Initial responses after tumor treatment have been reported to be correlated with overall survival (OS), and it has been suggested that initial responses may be used as a viable alternative for clinical response assessment (33-35). In our current study, a comparison of the TABE and DEB-TACE groups after PSM showed that the initial response and recurrence rate did not differ significantly between these two groups, which was consistent with the literature (36). The multivariate analysis showed that tumor size was an independent predictor of ORR (OR: 3.312,
95\% CI: $0.152-5.944)$. In addition, number of lesions and BCLC stage affected ORR (OR: 1.653, 95\% CI: 0.462-6.361; OR: 1.286, 95\% CI: 0.089-5.956); however, ORR was not significantly correlated with the intervention technique (TABE or DEB-TACE). As an important tumor marker for PLC, AFP plays an important role in the diagnosis, treatment, and prognostic prediction of HCC $(37,38)$. We also found a significant decrease in serum AFP level at 1 month postoperatively in both the TABE and DEB-TACE groups, but the difference was not statistically significant between these two groups. No patient achieved CR in either the TABE group or DEB-TACE group. This is probably because the patients enrolled in this study had a large tumor burden (the maximum tumor diameter reached about $8 \mathrm{~cm}$ on average). Additionally, all the patients received only 1 session of embolization.

PES is the most common complication of TABE or TACE. The syndrome, which has symptoms of transient 

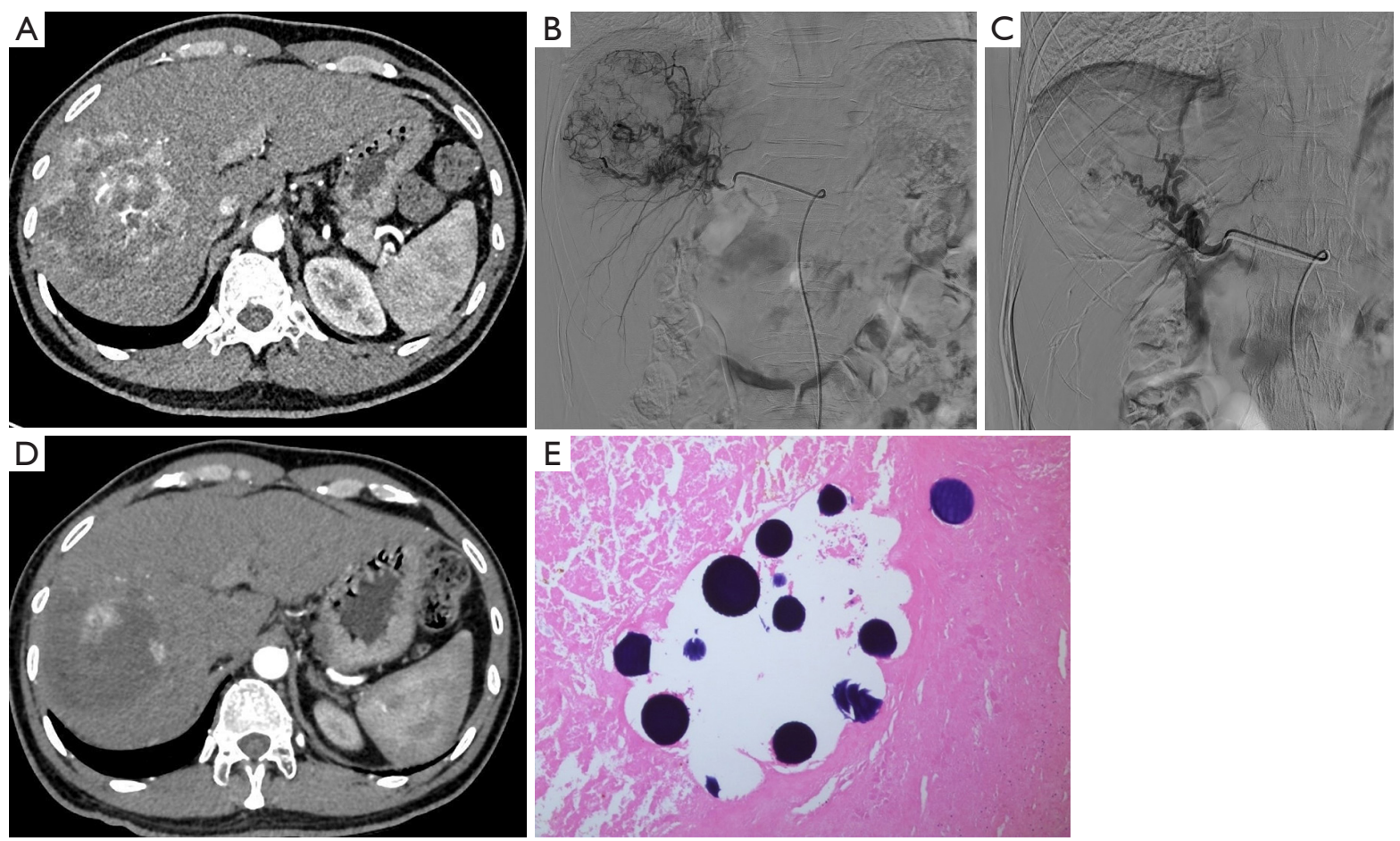

Figure 3 A 35-year-old male patient, with a previous history of hepatitis B for more than 10 years, presented at our center with vague upper right abdominal pain. (A) A CT scan revealed a mass in the right lobe of the liver in keeping with HCC; (B,C) a DEB-TACE was performed; (D) A CT scan performed 1 month after DEB-TACE revealed that most of the liver tumors were necrotic with residual contrast-enhanced tumor foci around them; and (E) surgical pathology confirmed that most of the tumor tissues were necrotic with embolic microspheres visible in the vessels $(\mathrm{H} \& \mathrm{E}$, original magnification $\times 200)$. HCC, hepatocellular carcinoma; DEB-TACE, drug-eluting beads-transarterial chemoembolization.

abdominal pain and fever, occurs in $60-80 \%$ of patients after TABE/TACE, and resolves spontaneously within 3-4 days (39). In the present study, PES symptoms, including fever, abdominal pain, and nausea/vomiting, were milder in the TABE group than the DEB-TACE group and were better tolerated in the TABE group. No serious adverse reactions, such as liver abscess, liver failure, or pancreatitis, occurred in either group after the intervention. In addition, the DEB-TACE group had a higher short-term risk of hepatotoxicity than the TABE group; however, there was no significant difference in hepatotoxicity between these two groups at 1 month postoperatively. No grade $3 / 4$ hepatotoxicity was observed, which suggests that TABE can effectively reduce short-term damage to normal liver tissue. Our findings are consistent with those reported in literature $(36,40)$. Thus, it can be concluded that TABE has significant advantages over DEB-TACE in reducing the adverse effects associated with hepatic cancer embolization.

The present study had a number of limitations. First, as a retrospective study, it had some inherent methodological weaknesses. Second, the patients were grouped according to their treatment protocols. This non-randomized design could lead to selection bias, and our conclusions on effectiveness and safety need to be further validated in prospective, multicenter, large-sample studies. Third, the present study showed that TABE had good safety and short-term efficacy in managing the spontaneous rupture of HCC, which paved the way for HCC to be treated repeatedly or to be managed with subsequent and systemic therapies; however, we only applied a 1-month follow-up period to assess the initial response of the tumors. Longerterm follow-up studies need to be conducted to further investigate the best response of tumors to TABE or DEBTACE and the OS of patients.

\section{Acknowledgments}

Funding: This work was supported by the Key-Area 
Research and Development Program of Guangdong Province, China (2020B010165004).

\section{Footnote}

Reporting Checklist: The authors have completed the STROBE reporting checklist. Available at https://dx.doi. org/10.21037/jgo-21-370

Data Sharing Statement: Available at https://dx.doi. org/10.21037/jgo-21-370

Conflicts of Interest: All authors have completed the ICMJE uniform disclosure form (available at https://dx.doi. org/10.21037/jgo-21-370). The authors have no conflicts of interest to declare.

Ethical Statement: The authors are accountable for all aspects of the work in ensuring that questions related to the accuracy or integrity of any part of the work are appropriately investigated and resolved. This multicenter retrospective propensity-score-matched non-randomized controlled study was approved by the Medical Ethics Committee of Shenzhen People's Hospital (No. LLKY-2021640). All procedures performed in this study involving human participants were in accordance with the Declaration of Helsinki (as revised in 2013). Informed consent has been attained from patients and his or her family before surgery, and informed consent for this retrospective study was waived.

Open Access Statement: This is an Open Access article distributed in accordance with the Creative Commons Attribution-NonCommercial-NoDerivs 4.0 International License (CC BY-NC-ND 4.0), which permits the noncommercial replication and distribution of the article with the strict proviso that no changes or edits are made and the original work is properly cited (including links to both the formal publication through the relevant DOI and the license). See: https://creativecommons.org/licenses/by-nc-nd/4.0/.

\section{References}

1. Sung H, Ferlay J, Siegel RL, et al. Global cancer statistics 2020: GLOBOCAN estimates of incidence and mortality worldwide for 36 cancers in 185 countries. CA Cancer J Clin 2021;71:209-49.

2. Miyamoto M, Sudo T, Kuyama T. Spontaneous rupture of hepatocellular carcinoma: a review of 172 Japanese cases. Am J Gastroenterol 1991;86:67-71.

3. Llovet JM, Kelley RK, Villanueva A, et al. Hepatocellular carcinoma. Nat Rev Dis Primers 2021;7:6. doi: 10.1038/ s41572-020-00240-3.

4. Lai EC, Lau WY. Spontaneous rupture of hepatocellular carcinoma: a systematic review. Arch Surg 2006;141:191-8.

5. Zhu LX, Wang GS, Fan ST. Spontaneous rupture of hepatocellular carcinoma. Br J Surg 1996;83:602-7.

6. Liu CL, Fan ST, Lo CM, et al. Management of spontaneous rupture of hepatocellular carcinoma: singlecenter experience. J Clin Oncol 2001;19:3725-32.

7. Leung KL, Lau WY, Lai PB, et al. Spontaneous rupture of hepatocellular carcinoma: conservative management and selective intervention. Arch Surg 1999;134:1103-7.

8. Cherqui D, Panis Y, Rotman N, et al. Emergency liver resection for spontaneous rupture of hepatocellular carcinoma complicating cirrhosis. Br J Surg 1993;80:747-9.

9. Ong GB, Taw JL. Spontaneous rupture of hepatocellular carcinoma. Br Med J 1972;4:146-9.

10. Kim JY, Lee JS, Oh DH, et al. Transcatheter arterial chemoembolization confers survival benefit in patients with a spontaneously ruptured hepatocellular carcinoma. Eur J Gastroenterol Hepatol 2012;24:640-5.

11. Bruix J, Sherman M. Management of hepatocellular carcinoma. Hepatology 2005;42:1208-36.

12. Department of Medical Administration, National Health and Health Commission of the People's Republic of China. Guidelines for diagnosis and treatment of primary liver cancer in China (2019 edition). Zhonghua Gan Zang Bing Za Zhi 2020;28:112-28.

13. European Association for Study of Liver; European Organisation for Research and Treatment of Cancer. EASL-EORTC clinical practice guidelines: management of hepatocellular carcinoma. Eur J Cancer 2012;48:599641. Erratum in: Eur J Cancer 2012;48:1255-6.

14. Lencioni R, Llovet JM. Modified RECIST (mRECIST) assessment for hepatocellular carcinoma. Semin Liver Dis 2010;30:52-60.

15. Kim BK, Kim SU, Kim KA, et al. Complete response at first chemoembolization is still the most robust predictor for favorable outcome in hepatocellular carcinoma. J Hepatol 2015;62:1304-10.

16. Zheng R, Qu C, Zhang S, et al. Liver cancer incidence and mortality in China: Temporal trends and projections to 2030. Chin J Cancer Res 2018;30:571-9.

17. Vergara V, Muratore A, Bouzari H, et al. Spontaneous rupture of hepatocelluar carcinoma: surgical resection and 
long-term survival. Eur J Surg Oncol 2000;26:770-2.

18. Chun YS, Pawlik TM, Vauthey JN. 8th Edition of the AJCC Cancer Staging Manual: Pancreas and Hepatobiliary Cancers. Ann Surg Oncol 2018;25:845-7.

19. Subramaniam S, Kelley RK, Venook AP. A review of hepatocellular carcinoma (HCC) staging systems. Chin Clin Oncol 2013;2:33.

20. Yang H, Chen K, Wei Y, et al. Treatment of spontaneous ruptured hepatocellular carcinoma: A single-center study. Pak J Med Sci 2014;30:472-6.

21. Kudo M, Chung H, Osaki Y. Prognostic staging system for hepatocellular carcinoma (CLIP score): its value and limitations, and a proposal for a new staging system, the Japan Integrated Staging Score (JIS score). J Gastroenterol 2003;38:207-15.

22. Llovet JM, Brú C, Bruix J. Prognosis of hepatocellular carcinoma: the BCLC staging classification. Semin Liver Dis 1999;19:329-38.

23. Corr P, Chan M, Lau WY, et al. The role of hepatic arterial embolization in the management of ruptured hepatocellular carcinoma. Clin Radiol 1993;48:163-5.

24. Shimada R, Imamura H, Makuuchi M, et al. Staged hepatectomy after emergency transcatheter arterial embolization for ruptured hepatocellular carcinoma. Surgery 1998;124:526-35.

25. Lee K, Qian DZ, Rey S, et al. Anthracycline chemotherapy inhibits HIF-1 transcriptional activity and tumor-induced mobilization of circulating angiogenic cells. Proc Natl Acad Sci U S A 2009;106:2353-8.

26. Arora AS, de Groen PC, Croall DE, et al. Hepatocellular carcinoma cells resist necrosis during anoxia by preventing phospholipase-mediated calpain activation. J Cell Physiol 1996;167:434-42.

27. Maluccio MA, Covey AM, Porat LB, et al. Transcatheter arterial embolization with only particles for the treatment of unresectable hepatocellular carcinoma. J Vasc Interv Radiol 2008;19:862-9.

28. Covey AM, Maluccio MA, Schubert J, et al. Particle embolization of recurrent hepatocellular carcinoma after hepatectomy. Cancer 2006;106:2181-9.

29. Bruix J, Llovet JM, Castells A, et al. Transarterial embolization versus symptomatic treatment in patients with advanced hepatocellular carcinoma: results of a randomized, controlled trial in a single institution. Hepatology 1998;27:1578-83.

30. Cammà C, Schepis F, Orlando $\mathrm{A}$, et al. Transarterial chemoembolization for unresectable hepatocellular carcinoma: meta-analysis of randomized controlled trials.
Radiology 2002;224:47-54.

31. Murata Y, Hiramatsu K, Yoshida Y, et al. Reactivation of intraabdominal tuberculous lymphadenopathy after drugeluting beads transcatheter arterial chemoembolization in a patient with hepatocellular carcinoma. Clin J Gastroenterol 2019;12:76-81.

32. Reid E, Suneja G, Ambinder RF, et al. Cancer in People Living With HIV, Version 1.2018, NCCN Clinical Practice Guidelines in Oncology. J Natl Compr Canc Netw 2018;16:986-1017.

33. Memon K, Kulik L, Lewandowski RJ, et al. Radiographic response to locoregional therapy in hepatocellular carcinoma predicts patient survival times. Gastroenterology 2011;141:526-35, 535.e1-2.

34. Malagari K, Alexopoulou E, Chatzimichail K, et al. Transcatheter chemoembolization in the treatment of HCC in patients not eligible for curative treatments: midterm results of doxorubicin-loaded DC bead. Abdom Imaging 2008;33:512-9.

35. Varela M, Real MI, Burrel M, et al. Chemoembolization of hepatocellular carcinoma with drug eluting beads: efficacy and doxorubicin pharmacokinetics. J Hepatol 2007;46:474-81.

36. Facciorusso A, Bellanti F, Villani R, et al. Transarterial chemoembolization vs bland embolization in hepatocellular carcinoma: A meta-analysis of randomized trials. United European Gastroenterol J 2017;5:511-8.

37. Sauzay C, Petit A, Bourgeois AM, et al. Alpha-foetoprotein (AFP): A multi-purpose marker in hepatocellular carcinoma. Clin Chim Acta 2016;463:39-44.

38. Riaz A, Ryu RK, Kulik LM, et al. Alpha-fetoprotein response after locoregional therapy for hepatocellular carcinoma: oncologic marker of radiologic response, progression, and survival. J Clin Oncol 2009;27:5734-42.

39. Wigmore SJ, Redhead DN, Thomson BN, et al. Postchemoembolisation syndrome--tumour necrosis or hepatocyte injury? Br J Cancer 2003;89:1423-7.

40. Meyer T, Kirkwood A, Roughton M, et al. A randomised phase II/III trial of 3-weekly cisplatin-based sequential transarterial chemoembolisation vs embolisation alone for hepatocellular carcinoma. Br J Cancer 2013;108:1252-9.

Cite this article as: Guo J, Wang W, Zhang Y, Xu L, Kong J. Comparison of initial tumor responses to transarterial bland embolization and drug-eluting beads-transarterial chemoembolization in the management of hepatocellular carcinoma: a propensity-score matching analysis. J Gastrointest Oncol 2021;12(4):1838-1850. doi: 10.21037/jgo-21-370 